\title{
Intranasal Ectopic Tooth - A Rare Case Report
}

\author{
Krishna Prasad Koirala, ${ }^{1}$ Bikram Babu Karki, ${ }^{1}$ Manita Maharjan ${ }^{1}$ \\ ${ }^{1}$ Department of ENT, Head and Neck, Manipal College of Medical Sciences, Pokhara, Nepal.
}

\begin{abstract}
Presence of ectopic tooth in the nasal cavity of an adult is a rare phenomenon. Exact etiology of this condition is yet to be confirmed. We report a case of a 40-year-oldwoman, who presented to us with the history of left nasal obstruction of one-year duration along with pain in the left side of face revealing an ectopic intra-nasal tooth on examination. Intranasal tooth was removed by surgical intervention with Luc's forceps. The patient's nasal obstruction and facial pain subsided after the treatment. We need to suspect an intranasal tooth when there is a white substance mimicking bone in the nasal cavity in patients presenting to us with facial pain and nasal obstruction
\end{abstract}

Keywords: intranasal tooth; nasal obstruction; surgical extraction.

\section{INTRODUCTION}

Ectopic eruption of tooth refers to the eruption of a tooth outside its normal position in the dental arch. The prevalence of ectopic canine tooth is reported as $5.5 \%$ in the literature. ${ }^{1}$ Most of the times it will not be noticed by the patient. It is found incidentally when the patient presents to the hospital with other symptoms pertaining to the area where it is present. It may be asymptomatic or may present as chronic or recurrent sinusitis, facial numbness, sepsis etc. ${ }^{2}$ However, ectopic eruption of teeth into the nasal cavity is a rare clinical entity. ${ }^{3}$ The cause of nasal tooth is unclear. Proposed theories are developmental disturbances such as cleft lip and palate, trauma or cystic lesions leading to tooth displacement, genetic factors, persistent deciduous teeth, and supernumerary teeth. ${ }^{4}$

The intranasal tooth maybe completely asymptomatic and may only be noted on routine clinical or radiographic examination. The diagnosis of nasal tooth is made on the basis of clinical and radiographic findings. Computed tomographic scanning is helpful in diagnosing and treatment planning. Chain et $\mathrm{al}^{5}$ reported three cases of ectopic teeth, 2 in the nasal cavity and one in the palate and found that nasal obstruction, purulent nasal discharge and nasal masses were the common presentations. Clinically the teeth were seen as white mass covered by the granulation tissue.

We are reporting a middle-aged woman, with a diagnosis of ectopic tooth in the nasal cavity, who presented to us with a one-year history of left nasal obstruction with features of allergic rhinitis. The objective of emphasizing the fact is that we should think of ectopic tooth when we see a white mass in the nasal cavity that mimics bare bone.

\section{CASE REPORT}

A 40-year-old female patient presented to our outpatient Department with left nasal obstruction, watery nasal discharge, and excessive sneezing of 1 -year duration. There was no history of headache, facial pain and numbness, loosening of tooth, cleft palate surgery, surgical or physical trauma. There was no similar history in any of her family members. She had visited local medical shop for her problem and referred to us as there was no symptomatic relief. Her general condition was normal. On examination of the nose, there was decreased patency on the left side. Anterior rhinoscopy revealed left sided simple deviated nasal septumwith right sided inferior turbinate hypertrophy. On further examination, there was a shiny white mass in the floor of left nasal cavity below and medial to left inferior turbinate. On probing, it was fixed, immobile and bony hard in consistency. There was no bleeding on probing, bad smell, and no loosening of teeth on oral cavity examination. Nasolabial area was normal on palpation. Oral cavity examination was found to be normal with 14 upper teeth and 16 lower teeth. There were no loose teeth. Rest of the otolaryngology examination was unremarkable. Her routine blood and urine examination were within normal limits.

Computerized Tomography (CT) scan of nose and paranasal sinuses (PNS), plain, coronal cuts was performed to identify the nature of bony mass in the left nasal cavity. CT scan showed DNS to left

Correspondence: Dr. Krishna Prasad Koirala, Department of ENT and Head and Neck Surgery, Manipal College of Medical Sciences, Pokhara, Nepal. Email: gaukrishna9@gmail.com. Phone: +977-9856034347. Article received: 2020-04-27. Article accepted: 2020-06-14. 
Koirala et al. Intranasal Ectopic Tooth - A Rare Case Report.

with right inferior turbinate hypertrophy with radiopaque dense mass with bony consistency in left nasal cavity with bilateral maxillary and ethmoidal sinus mucosal thickening (Figure 1).

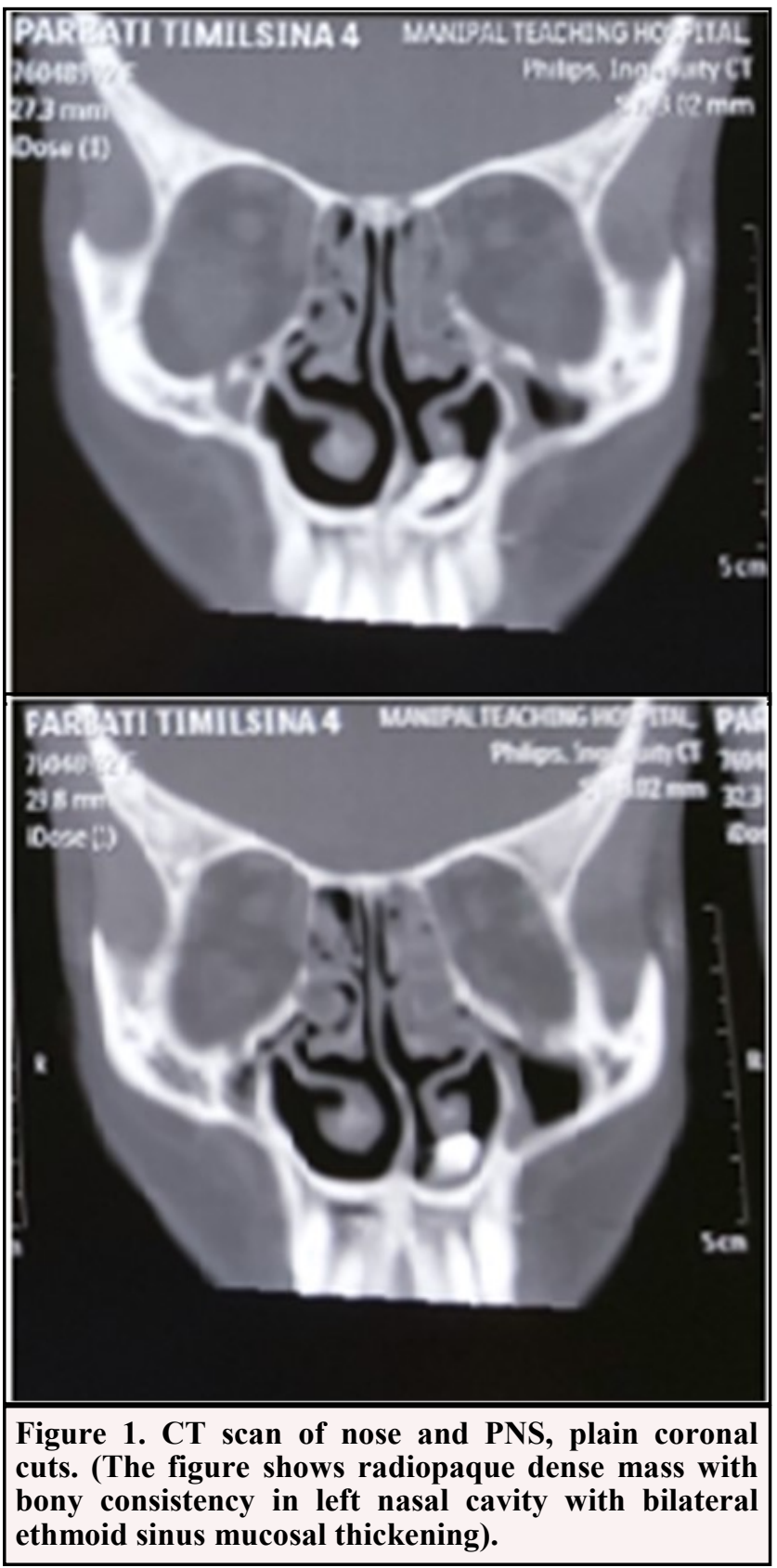

She underwent removal of intranasal tooth with Luc's forceps and septoplasty with bilateral partial inferior turbinectomy under general anesthesia. Removed tooth is shown in figure. 2. Both nasal cavities were packed with merocel which was removed after 72 hours. After pack removal, her left nasal cavity became patent and patient was discharged home.

Patient was called for follow up after 2 weeks of surgery. There was no nasal obstruction and facial pain. Septoplasty wound was healing, and patient was comfortable. Histological study confirmed the dental nature of the mass.
Figure 2. Removed ectopic tooth.

Patient was followed up after a month. The wound had almost healed and there was no recurrence of tooth (Figure. 3).

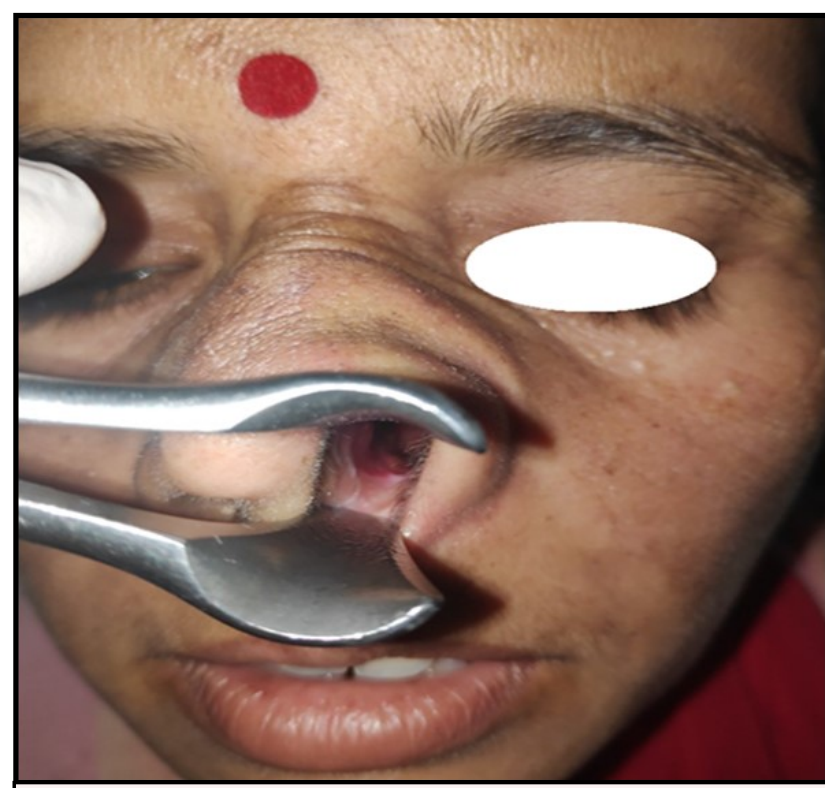

Figure 3. Post-operative photograph after a month. Wound has healed and there is no recurrence of tooth.

\section{DISCUSSIONS}

Intranasal teeth are a rare presentation of ectopic teeth with varied manifestation. They are typically associated with cleft lip and alveolus in children. Mulhim et al ${ }^{6}$ reported an intranasal tooth in a 11year-old girl who presented with unilateral nasal obstruction and occasional headache without any obvious etiology. Our patient also presented with the similar clinical finding. The nasal fossa is one of several sites in which ectopic teeth may be found. ${ }^{7}$ Unilateral nasal obstruction is a common complaint, but it can be an incidental finding during routine examination in patients without nasal discomfort.

Levin et $\mathrm{al}^{7}$ conducted a systematic review in ectopic sinonasal teeth in 23 articles and reported that 
nasal obstruction (48.14\%), rhinorrhea (22.2\%), facial pain $(22.2 \%)$ and epistaxis $(22.2 \%)$ to be the most common presentations. Simple extraction under general anesthesia was sufficient in $41 \%$ of patients in their study. We also performed the surgery under general anesthesia.

Clinically, an intranasal tooth may be seen as a hard-white mass in the nasal cavity surrounded by debris. The differential diagnosis includes foreign body, rhinolith, inflammatory lesion with calcification calcified polyp, osteoma, and malignant tumors like osteosarcoma and chondrosarcoma. However, CT scan is confirmatory of the diagnosis. ${ }^{8}$ We also suspected the same in CT scan of Nose and PNS.

Standard treatment for an ectopic tooth is extraction, but asymptomatic ectopic tooth can be kept under

\section{REFERENCES}

1. Jain S, Debbarma S. Patterns and prevalence of canine anomalies in orthodontic patients. Med Pharm Rep. 2019 Jan; 92(1): 72-8.PMID: 30957090

2. Lombroni L G, Farronato G, Santamaria G, Lombroni D M, Gatti P, Capelli M. Ectopic teeth in the maxillary sinus: A case report and literature review.Indian J Dent Res. Sep-Oct 2018;29(5):667-71. doi: 10.4103/ ijdr.IJDR_347_17.

3. Yeung $\mathrm{K} \overline{\mathrm{H}}$, Lee $\mathrm{KH}$. Intranasal tooth in apatent with a clef lip and alveolus. Cleft Palate Craniofac J. 1996 Mar;33(2):157-9. doi: $10.1597 / 1545-$

156919960330157 itiapw 2.3.co 2.

4. GuptaY K,Shah̄N. Intranasal tooth as a complication of cleft lip and alveolus in a fouryear-old child: case report and literature review.Int J Paediatr Dent. 2001 May;11(3):221 $-4$.

5. A Chen, Huang JK, Cheng SJ, Sheu CY. Nasal Teeth: Report of Three Cases. American Journal of Neuroradiology April 2002;23 continued observation also. However, complications like septal abscess, nasolacrimal duct obstruction, chronic foul-smelling nasal discharge, septal perforation, oro-nasal fistula bacterial and fungal sinusitis etc. have been reported with long standing ectopic tooth in the nose. ${ }^{9-10}$ However there is no mention of cancer and recurrent ectopic tooth in adults in the literature.

\section{CONCLUSION}

Ectopic tooth in the nasal cavity is to be suspected when hard, fixed bone like lesion is seen in the nasal floor. Early extraction helps to avoid infection and other complications.

Citation: Koirala KP, Karki BB, Maharjan M. Intranasal Ectopic Tooth - A Rare Case Report. JCMS Nepal. 2020; 16(2):119-21. 